\title{
A Review of the Incidence and Management of New- Onset Atrial Fibrillation Post Transcatheter Aortic Valve Implantation
}

\author{
Supna Saxena ${ }^{1 *}$ and Daryoush Javidi ${ }^{2}$ \\ ${ }^{1}$ Medical Student, California University of Science and Medicine, USA \\ ${ }^{2}$ Associate Professor of Medical Education \& Clinical Skills, California University of Science and Medicine, USA
}

*Corresponding author: Supna Saxena, Medical Student, California University of Science and Medicine, 217 East Club Center Drive, Suite A, San Bernardino, CA 92408, USA.

To Cite This Article: Supna Saxena, A Review of the Incidence and Management of New-Onset Atrial Fibrillation Post Transcatheter Aortic Valve Implantation. 2020 - 8(2). AJBSR.MS.ID.001251. DOI: 10.34297/AJBSR.2020.08.001251.

Received: 眥 March 03, 2020; Published: 㘹 March 16, 2020

\begin{abstract}
Transcatheter Aortic Valve Implantation (TAVI) is an established treatment option for patients with aortic stenosis who are considered highrisk for surgical valve replacement. New-onset atrial fibrillation (NOAF) is a serious adverse event post TAVI that is predictive of poor outcomes and whose pathophysiology remains unclear. The incidence of NOAF post-TAVI has a reportedly broad range depending on the study but has been correlated to procedure approach, choice of valve, and the use of general anesthesia. While surgical aortic valve replacement (SAVR) has traditionally been the preferred treatment for aortic stenosis, recent studies have demonstrated the benefits of expanding TAVI into a larger patient population. Additionally, the management of NOAF post-TAVI remains highly variable. This review paper aims to summarize the incidence of NOAF and to outline management strategies.

The risk factors for NOAF post-TAVI is an ongoing area of research with many questions remaining to be answered. With the expansion of TAVI into lower-risk patient populations, it is imperative that risk factors for NOAF be determined in order to decrease the likelihood of stroke and mortality after the procedure. Data is still limited on standardized treatment for NOAF, but multiple international drug trials are aiming to discern the optimal anti-platelet and anti-coagulation treatment to improve post-procedure outcomes.
\end{abstract}

Keywords: Transcatheter Aortic Valve Implantation (TAVI); Atrial Fibrillation; Aortic Stenosis

Abbreviations: TAVI: Transcatheter Aortic Valve Implantation; AF: Atrial Fibrillation; NOAF: New-Onset Atrial Fibrillation; SAVR: Surgical Aortic Valve Replacement; SAPT: Single Antiplatelet Therapy; DAPT: Double Antiplatelet Therapy; OAC: Oral Anticoagulation; DOAC: Direct Oral Anticoagulation

\section{Introduction}

Transcatheter aortic valve implantation (TAVI) is a relatively new procedure for aortic stenosis that has been traditionally reserved for higher risk patients with the alternative being surgical valve replacement [1]. Prior to the first FDA approval of TAVI in 2011, the alternative to surgical valve replacement was palliative care via balloon valvuloplasty and diuretics [2]. Since its approval for high-risk patients, the long-term outcomes for patients undergoing TAVI have continued to improve [3]. While TAVI is becoming a more popular treatment option, the 5-year mortality can be as high as $59.1 \%$ with predictors including atrial fibrillation (AF) and access route for TAVI [4]. AF is common in high-risk patients with severe aortic stenosis and is associated with an increased risk of bleeding complications and all-cause mortality [5-7].

The pathophysiology of AF remains unclear. Recent studies have attempted to elucidate the underlying cause and findings suggest a mechanistic link between inflammatory processes and the development of AF. Factors such as C-reactive protein and specific gene mutations have been seen in the presence of $\mathrm{AF}$ and as a predictor of developing future AF [8]. This inflammatory response may be a possible explanation for the increased incidence 
of AF in surgical aortic valve replacement (SAVR) and non-femoral TAVI patients [9]. Findings from the SOURCE XT Trial suggest that the presence of either pre-existing or new-onset atrial fibrillation (NOAF) increased all-cause and cardiac mortality [10]. Studies report a wide range of incidence rates of NOAF and this variability is partly due to a lack of a consistent definition. However, the majority of studies define NOAF as someone with no history of atrial fibrillation prior to the procedure and who had an episode lasting greater than 30 seconds post-TAVI $[1,11]$. The incidence of NOAF post-TAVI ranges from $6.0 \%$ to $50.7 \%$ with an increased incidence in patients with larger left atrium size and those undergoing transapical TAVI [11-14]. Patients with NOAF post-TAVI have shown an increased risk of cardiovascular events and stroke, however studies are divided on whether or not NOAF leads to a higher rate of all-cause mortality [7,10-17]. We aim to summarize the incidence and risk factors of NOAF post-TAVI and outline the different available management strategies.

\section{Incidence of NOAF Post-TAVI vs. Surgical Aortic Valve Replacement}

When TAVI was approved as a treatment for aortic stenosis it was intended as a treatment option for high-risk patients for whom surgical aortic valve replacement (SAVR) was not an option. However, recent studies have consistently shown that in terms of NOAF, TAVI has better outcomes compared to SAVR $[1,18]$. Motloch et al. [1] conducted one of the first studies comparing patient outcomes after undergoing TAVI or SAVR. In a sample of 170 patients, they found that there was a lower incidence of NOAF in the TAVI group compared to the SAVR group (3.5\% vs. 30.7\%) and that NOAF only occurred in the TAVI group when the transapical approach was used. However, one caveat of the study is that they defined NOAF as an episode occurring post TAVI that lasted at least 10 minutes. Since this duration is longer than the more common cut off of 30 seconds, it is possible that the incidence in the TAVI group was underestimated [1]. Tanawuttiwat et al. [19] replicated these results in a population of 231 patients, 149 of which underwent TAVI. The total incidence of NOAF in the TAVI group was $20.8 \%$ compared to an incidence of $60 \%$ in the SAVR group. When divided into subgroups, they demonstrated a significant difference between the incidence of NOAF in the SAVR group and transfemoral TAVI group, but a similar incidence of NOAF between the SAVR group and the transapical TAVI group [19]. In a more recent study, Kalra et al. conducted an analysis of 171,480 hospitalizations for TAVI or SAVR. They found that the incidence of NOAF was comparable between TAVI patients and patients undergoing SAVR and that NOAF in both cases had higher odds of in-hospital mortality compared with those without NOAF [20].

While initially thought of as a treatment option only for high-risk patients, TAVI has slowly been shown to be an effective treatment option for intermediate risk patients. Sondergaard et al. [21] conducted an analysis of 280 patients in the NORDIC trial that were randomized to either the TAVI or SAVR group. This study was the first to document that there was no significant difference between the TAVI and SAVR groups of all-cause mortality in patients with a lower surgical risk [21]. The PARTNER 2 Trial also demonstrated the non-inferiority of TAVI compared to SAVR in intermediate-risk patients. The incidence of NOAF specifically was lower in the TAVI group compared to SAVR [22]. Sardar et al. [23] conducted a metaanalysis of intermediate-risk aortic stenosis patients which showed that TAVI had a lower rate of NOAF compared to SAVR as well as lower rates of kidney injury and major bleeding, demonstrating safety and efficacy in intermediate-risk patients [23]. These findings encourage the expansion of TAVI as the preferred treatment option in lower-risk aortic stenosis patients. Among patients with severe aortic stenosis who were at lower surgical risk, the rate of mortality, stroke, and rehospitalization at 1 year was significantly lower with TAVI than with surgery [24]. Recent studies suggest that transfemoral TAVI with newer generation heart valves and delivery systems might even be superior to traditional surgical valve replacement in high and intermediate-risk patients in regards to all-cause mortality and stroke, making TAVI the first line therapy for severe aortic stenosis irrespective of surgical risk [25]. Indeed, there have been reports of a dramatic decrease in 1-year mortality and adverse events in recent years in patients undergoing SAVR for severe aortic stenosis that is attributed to the selection of increasingly lower-risk patients for surgery now that TAVI is presented as an alternative treatment option [26]. As TAVI methodology continues to improve, it is emerging as the superior medical therapy in terms of mortality for extreme-risk aortic stenosis patients, non-inferior or superior to surgery in high-risk patients, and non-inferior to surgery in intermediate risk patients. However, while transfemoral TAVI has been shown to have a lower incidence of NOAF compared to SAVR, there are some limitations that affect outcomes post-TAVI $[27,28]$.

\section{Risk factors of NOAF Post-TAVI}

Since TAVI has traditionally been the treatment of choice in higher risk populations, it is difficult to elucidate the risk factors unique to NOAF when many patients have co-morbidities. However, a pattern exists in the literature showing the protective nature of transfemoral TAVI compared to the transapical approach [1]. Transfemoral access was shown to result in a lower rate of all-cause mortality and stroke even compared to surgery [14,22]. Specifically, the transapical approach is an independent predictor of NOAF post TAVI [19,20, 29,30]. When separating apart the different TAVI approaches, Tanawuttiwat et al. [19] found that NOAF was least common when using the transfemoral approach and most common when using the transapical approach [19]. Additionally, of the TAVI approaches, only the transapical approach had a higher incidence of major and life-threatening bleeding [13]. It has been 
proposed that transapical patients who were most at risk of cardiac complications included those who remained intubated and under intravenous inotropic support during the postprocedural phase, patients who receive preprocedural oral inotropes, or patients with pacemaker rhythm at baseline [31]. However, it should be noted that the transapical route for TAVI is mostly used in patients in whom access to the femoral artery is limited [30]. Therefore, it is difficult to determine if these patients who go through transapical TAVI are inherently more pre-disposed to developing NOAF or if it is an element of the procedure itself that is an instigating factor. It has been observed that there is a lesser degree of inflammation in the transfemoral procedure compared to the transapical which would provide a potential explanation for the discrepancy in the incidence of NOAF [32].

Apart from access route, studies have demonstrated several other factors associated with NOAF post-TAVI. While it does not seem that gender or co-morbid diabetes mellitus affects the incidence of NOAF post-TAVI, there is a positive correlation with prosthetic valve size, general anesthesia and age [20,30,33-35]. There was also a statistically significant association with NOAF and a history of chronic pulmonary disease at baseline [20,36,37]. Additionally, several authors noted a difference in the incidence of NOAF between using the balloon-expandable Edwards SAPIEN valve and the self-expandable Medtronic CoreValve. The Edwards SAPIEN valve was associated with NOAF in $31.9 \%$ of patients [11] while another study observed a lower incidence of $7.7 \%$ with the Medtronic CoreValve [38]. The significance of this observation cannot be determined as these results were published in two different studies with numerous confounding factors, however it is a trend repeated several times in the literature. When both valves were used in the same study, there was a higher incidence of NOAF when using the Edwards SAPIEN valve compared to the Edwards SAPIEN XT or the CoreValve [35]. Additionally, balloon post-dilation was shown to be an independent risk factor for NOAF, which is a quality unique to the Edwards SAPIEN valves as the Medtronic CoreValves are self-expandable. Balloon expandable valves had an incidence of NOAF of $69.7 \%$ compared to an incidence of $30.3 \%$ when using a self-expandable valve [39]. However, a meta-analysis conducted by Sannino et al. [14] demonstrated that there were no significant differences between the SAPIEN valve and CoreValve in terms of the incidence of NOAF [14]. Although a major drawback of this study is that it only looked at five different studies with a total of 4,604 patients. Ideally, a clinical trial unaffiliated with either company should be conducted that directly compares these two valves in the setting of NOAF post TAVI.

\section{Management}

Patients who undergo TAVI are typically older and at higher surgical risk and accordingly, have more co-morbidities to consider when developing management strategies. Limited data are available regarding the optimal treatment plan for these patients who also need therapy for NOAF $[40,41]$. The different strategies consist of either single antiplatelet therapy (SAPT) or double antiplatelet therapy (DAPT), or oral anticoagulation therapy (OAC) [42]. When there is no known indication for oral anticoagulation, such as pre-existing atrial fibrillation, SAPT is preferred to DAPT to minimize bleeding risk $[42,43]$. However, other studies have contradicted this recommendation by showing that patients with NOAF discharged with SAPT showed a higher rate of mortality and that this population specifically would benefit from a more aggressive antithrombotic therapy [39]. There is no clear optimal antithrombotic therapy for TAVI patients with baseline or new AF but triple therapy with OAC and DAPT is to be avoided $[44,45]$. In the context of bioprosthetic valve thrombosis, OAC has shown to be more effective compared to DAPT [44]. AF is considered an indication for OAC, which is believed to be more effective than DAPT or SAPT in terms of stroke prevention [42]. OAC therapy has been shown to be safe in both patients with baseline $\mathrm{AF}$ and those who develop NOAF post-TAVI, in that OAC did not result in excess risk of bleeding or stroke compared to DAPT. Additionally, DAPT in these patients were shown to increase mortality [46]. Conversely, patients who developed NOAF post TAVI and who were not prescribed anticoagulation therapy were at the highest risk of adverse effects [45].

While OAC therapy typically consists of a Vitamin K Antagonist (VKA) such as warfarin, studies have started to investigate the efficacy of novel direct oral anticoagulants (DOAC) in the treatment of AF post TAVI. While treatment with continuous VKA did not show any increase in safety, treatment with DOAC was associated with a significantly reduced rate of 1-year mortality [47]. One example of a DOAC is rivaroxaban, a direct factor Xa inhibitor and the most commonly used non VKA anticoagulant. In the ROCKETAF Trial, rivaroxaban was shown to be non-inferior to warfarin in terms of stroke prevention and had superior rates of drug adherence compared to warfarin [48]. With this data in mind, the GALILEO Trial was started to compare a rivaroxaban-based antithrombotic strategy to an antiplatelet-based strategy after TAVI [49]. Windecker et al. [50] described the trial design of the GALILEO trial where patients were randomized with the trial group taking $10 \mathrm{mg}$ rivaroxaban once daily with ASA for 90 days followed by only rivaroxaban thereafter [50]. Unfortunately, this Phase III trial was terminated in 2018 because the trial arm had higher rates of death and adverse events compared to the traditional anti-platelet group in preliminary data.

Alternatively, researchers have been investigating apixaban as another DOAC to be used instead of warfarin. In the ARISTOTLE Trial, apixaban was shown to be superior to warfarin in patients with $\mathrm{AF}$ in preventing stroke and in reducing bleeding events [51]. 
Seeger et al. [51] demonstrated that patients with AF taking VKA presented with higher rates of acute kidney stages II and III and there was a lower stroke rate with apixaban compared to VKA after 30 days [51]. These findings led to the ongoing randomized ATLANTIS Trial looking at the superiority of apixaban compared to traditional VKA therapy in patients following TAVI [52]. While this study is anticipated to end in 2020, patients with NOAF were not included in the experimental arm of the trial due to safety reasons.

\section{Conclusion}

The risk factors for NOAF post-TAVI is an ongoing area of research with many questions remaining to be answered. With the expansion of TAVI into lower-risk patient populations, it is imperative that risk factors for NOAF be determined in order to decrease the likelihood of stroke and mortality after the procedure. Of these risk factors, studies should investigate the mechanism that results in the transapical approach significantly increasing the risk of NOAF. Additionally, there is a correlation between balloonexpandable valves and greater rates of NOAF, but studies directly comparing these two valves have not yet been conducted. It may be hard to obtain unbiased data in this regard as these companies provide much of the funding for these post-TAVI studies. TAVI has shown to be non-inferior to SAVR and in terms of incidence of NOAF, has been shown in multiple studies to be superior. This observation combined with the continuous improvement of valves and methodology indicate that TAVI will likely become the firstline option for the treatment of severe aortic stenosis. Data is still limited on standardized treatment for NOAF, but there are ongoing drug trials that are aiming to discern the optimal anti-platelet and anti-coagulation treatment to improve post-procedure outcomes. As the pathophysiology of NOAF post TAVI remains unclear, it would be beneficial for future randomized clinical trials to look at this population of patients specifically as the standard of care may have different outcomes on stroke and mortality.

\section{Disclosure of Conflict of Interest}

There are no conflicts of interest to declare by any of the authors of this study.

\section{References}

1. Motloch LJ, Reda S, Rottlaender D, Khatib R, Müller Ehmsen J, et al (2012) Postprocedural atrial fibrillation after transcatheter aortic valve implantation versus surgical aortic valve replacement. Ann Thorac Surg 93(1): 124-131.

2. Cribier A (2016) The development of transcatheter aortic valve replacement (TAVR). Glob Cardiol Sci Pract 2016(4): e201632.

3. Ludman PF, Moat N, de Belder MA, Blackman DJ, Duncan A, et al. (2015) Transcatheter aortic valve implantation in the United Kingdom: temporal trends, predictors of outcome, and 6-year follow-up: a report from the UK Transcatheter Aortic Valve Implantation (TAVI) Registry, 2007 to 2012. Circulation 131(13): 1181-90.

4. Zahn R, Werner N, Gerckens U, Linke A, Sievert H, et al. (2017) Five-year follow-up after transcatheter aortic valve implantation for symptomatic aortic stenosis. Heart 103(24): 1970-1976.
5. Herold J, Herold Vlanti V, Sherif M, Luani B, Breyer C, et al. (2017) Analysis of cardiovascular mortality, bleeding, vascular and cerebrovascular events in patients with atrial fibrillation vs. sinus rhythm undergoing transfemoral Transcatheter Aortic Valve Implantation (TAVR). BMC Cardiovasc Disord 17(1): 298.

6. Stortecky S, Buellesfeld L, Wenaweser P, Heg D, Pilgrim T, et al. (2013) Atrial fibrillation and aortic stenosis: impact on clinical outcomes among patients undergoing transcatheter aortic valve implantation. Circ Cardiovasc Interv 6(1): 77-84.

7. Biviano AB, Nazif T, Dizon J, Garan H, Fleitman J, et al. (2016) Atrial Fibrillation Is Associated with Increased Mortality in Patients Undergoing Transcatheter Aortic Valve Replacement: Insights from the Placement of Aortic Transcatheter Valve (PARTNER) Trial. Circ Cardiovasc Interv 9(1).

8. Engelmann MD, Svendsen JH (2005) Inflammation in the genesis and perpetuation of atrial fibrillation. Eur Heart J 26(20): 2083-2092.

9. Rettig TCD, Nijenhuis VJ, Meek B, Rigter S, Ten Berg JM, et al. (2018) Systemic Inflammation after Transcatheter Aortic Valve Implantation: A Prospective Exploratory Study. J Cardiothorac Vasc Anesth 32(4): e77-e82.

10. Tarantini G, Mojoli M, Windecker S, Wendler O, Lefèvre T, et al. (2016) Prevalence and Impact of Atrial Fibrillation in Patients with Severe Aortic Stenosis Undergoing Transcatheter Aortic Valve Replacement: An Analysis from the SOURCE XT Prospective Multicenter Registry. JACC Cardiovasc Interv 9(9): 937-946.

11. Amat-Santos IJ, Rodés Cabau J, Urena M, DeLarochellière R, Doyle D, et al. (2011) Incidence, predictive factors, and prognostic value of new-onset atrial fibrillation following transcatheter aortic valve implantation. J Am Coll Cardiol 59(2): 178-188.

12. Yoon YH, Ahn JM, Kang DY, Ko E, Lee PH, et al. (2019) Incidence, Predictors, Management, and Clinical Significance of New-Onset Atrial Fibrillation After Transcatheter Aortic Valve Implantation. Am J Cardiol 123(7): 1127-1133.

13. Furuta A, Lellouche N, Mouillet G, Dhanjal T, Gilard M, et al. (2016) Prognostic value of new onset atrial fibrillation after transcatheter aortic valve implantation: A FRANCE 2 registry substudy. Int J Cardiol 210: $72-79$.

14. Sannino A, Gargiulo G, Schiattarella GG, Perrino C, Stabile E, et al. (2016) A meta-analysis of the impact of pre-existing and new-onset atrial fibrillation on clinical outcomes in patients undergoing transcatheter aortic valve implantation. EuroIntervention 12(8): e1047-e1056.

15. Jørgensen TH, Thygesen JB, Thyregod HG, Svendsen JH, Søndergaard L (2015) New-onset atrial fibrillation after surgical aortic valve replacement and transcatheter aortic valve implantation: a concise review. J Invasive Cardiol 27(1): 41-47.

16. Yankelson L, Steinvil A, Gershovitz L, Leshem Rubinow E, Furer A, et al. (2014) Atrial fibrillation, stroke, and mortality rates after transcatheter aortic valve implantation. Am J Cardiol 114(12): 1861-1866.

17. Chopard R, Teiger E, Meneveau N, Chocron S, Gilard M, et al. (2015) Baseline Characteristics and Prognostic Implications of Pre-Existing and New-Onset Atrial Fibrillation After Transcatheter Aortic Valve Implantation: Results From the FRANCE-2 Registry. JACC Cardiovasc Interv 8(10): 1346-1355.

18. Jørgensen TH, Thyregod HG, Tarp JB, Svendsen JH, Søndergaard L (2017) Temporal changes of new-onset atrial fibrillation in patients randomized to surgical or transcatheter aortic valve replacement. Int J Cardiol (234): 16-21.

19. Tanawuttiwat T, O’Neill BP, Cohen MG, Chinthakanan O, Heldman AW, et al. (2014) New-onset atrial fibrillation after aortic valve replacement: comparison of transfemoral, transapical, transaortic, and surgical approaches. J Am Coll Cardiol 63(15): 1510-1519.

20. Kalra R, Patel N, Doshi R, Arora G, Arora P (2019) Evaluation of the Incidence of New-Onset Atrial Fibrillation After Aortic Valve Replacement. JAMA Intern Med 179(8): 1122-1130. 
21. Søndergaard L, Steinbrüchel DA, Ihlemann N, Nissen H, Kjeldsen BJ, et al. (2016) Two-Year Outcomes in Patients with Severe Aortic Valve Stenosis Randomized to Transcatheter Versus Surgical Aortic Valve Replacement: The All-Comers Nordic Aortic Valve Intervention Randomized Clinical Trial. Circ Cardiovasc Interv 9(6): e003665.

22. Leon MB, Smith CR, Mack MJ, Makkar RR, Svensson LG, et al. (2016) PARTNER 2 Investigators. Transcatheter or surgical aortic-valve replacement in intermediate-risk patients. N Engl J Med 374(17): 16091620 .

23. Sardar P, Kundu A, Chatterjee S, Feldman DN, Owan T, et al. (2017) Transcatheter versus surgical aortic valve replacement in intermediaterisk patients: Evidence from a meta-analysis. Catheter Cardiovasc Interv 90(3): 504-515.

24. Mack MJ, Leon MB, Thourani VH, Makkar R, Kodali SK, et al. (2019) PARTNER 3 Investigators. Transcatheter aortic-valve replacement with a balloon-expandable valve in low-risk patients. N Engl J Med 380: 16951705.

25. O'Sullivan CJ, Wenaweser P (2017) A Glimpse into the Future: In 2020, Which Patients will Undergo TAVI or SAVR? Interv Cardiol 12(1): 44-50.

26. Rozen G, Fefer P, Shinfeld A, Sternik L, Guetta V, et al. (2015) The changing characteristics and outcomes of patients undergoing surgical aortic valve replacement in the transcatheter aortic valve implantation era. J Cardiovasc Med 16(4):261-266.

27. Amrane H, Deeb GM, Popma JJ, Yakubov SJ, Gleason TG, et al. (2018) Causes of death in intermediate-risk patients: The Randomized Surgical Replacement and Transcatheter Aortic Valve Implantation Trial. J Thorac Cardiovasc Surg 158(3): 718-728.e3.

28. De Biase C, Mastrokostopoulos A, Philippart R, Bonfils L, Berthoumieu $P$ et al. (2018) What are the remaining limitations of TAVi? J Cardiovasc Surg (Torino) 59(3): 373-380.

29. Maan A, Heist EK, Passeri J, Inglessis I, Baker J, et al. (2015) Impact of atrial fibrillation on outcomes in patients who underwent transcatheter aortic valve replacement. Am J Cardiol 115(2):220-226.

30. Koniari I, Tsigkas G, Kounis N, Velissaris D, Chourdakis E, et al. (2018) Incidence, pathophysiology, predictive factors and prognostic implications of new onset atrial fibrillation following transcatheter aortic valve implantation. J Geriatr Cardiol 15(1): 50-54.

31. Patris V, Giakoumidakis K, Argiriou M, Naka KK, Apostolakis E, et al. (2018) Factors associated with early cardiac complications following transcatheter aortic valve implantation with transapical approach. Pragmat Obs Res 9: 21-27.

32. Stahli BE, Grunenfelder J, Jacobs S, Falk V, Landmesser U, et al. (2012) Assessment of inflammatory response to transfemoral transcatheter aortic valve implantation compared to transapical and surgical procedures: a pilot study. J Invasive Cardiol 24(8): 407-411.

33. Ando T, Takagi H, Briasoulis A, Umemoto T (2017) Does diabetes mellitus impact prognosis after transcatheter aortic valve implantation? Insights from a meta-analysis. J Cardiol 70(5): 484-490.

34. Wang TY, Gracia E, Callahan S, Bilfinger T, Tannous H et al. (2019) Gender Disparities in Management and Outcomes Following Transcatheter Aortic Valve Implantation with Newer Generation Transcatheter Valves. Am J Cardiol 123(9): 1489-1493.

35. Barbash IM, Minha S, Ben-Dor I, Dvir D, Torguson R, et al. (2015) Predictors and clinical implications of atrial fibrillation in patients with severe aortic stenosis undergoing transcatheter aortic valve implantation. Catheter Cardiovasc Interv 85(3): 468-477.

36. Dziewierz A, Tokarek T, Kleczynski P, Sorysz D, Bagienski M, et al. (2018) Impact of chronic obstructive pulmonary disease and frailty on long-term outcomes and quality of life after transcatheter aortic valve implantation. Aging Clin Exp Res 30(9): 1033-1040.
37. Gupta T, Goel K, Kolte D, Khera S, Villablanca PA, et al. (2017) Association of chronic kidney disease with in-hospital outcomes of transcatheter aortic valve replacement. JACC Cardiovasc Interv 10(20): 2050-2060.

38. Popma JJ, Deeb GM, Yakubov SJ, Mumtaz M, Gada H, et al. (2019) Transcatheter Aortic-Valve Replacement with a Self-Expanding Valve in Low-Risk Patients. N Engl J Med 380(18): 1706-1715.

39. Sannino A, Stoler RC, Lima B, Szerlip M, Henry AC, et al. (2016) Frequency of and Prognostic Significance of Atrial Fibrillation in Patients Undergoing Transcatheter Aortic Valve Implantation. Am J Cardiol 118(10): 1527-1532.

40. Vavuranakis M, Kolokathis AM, Vrachatis DA, Kalogeras K, Magkoutis NA, et al. (2016) Atrial Fibrillation During or After TAVI: Incidence, Implications and Therapeutical Considerations. Curr Pharm Des 22(13): 1896-1903.

41. Vavuranakis M, Kalogeras K, Kolokathis AM, Vrachatis D, Magkoutis N, et al. (2018) Antithrombotic therapy in TAVI. J Geriatr Cardiol 15(1): 6675.

42. Nijenhuis VJ, Brouwer J, Søndergaard L, Collet JP, Grove EL, et al. (2019) Antithrombotic therapy in patients undergoing transcatheter aortic valve implantation. Heart 105(10): 742-748.

43. Valvo R, Costa G, Tamburino C, Barbanti M (2019) Antithrombotic Therapy in Transcatheter Aortic Valve Replacement. Front Cardiovasc 6: 73.

44. Sherwood MW, Vora AN (2018) Challenges in Aortic Stenosis: Review of Antiplatelet/Anticoagulant Therapy Management with Transcatheter Aortic Valve Replacement (TAVR): TAVR with Recent PCI, TAVR in the Patient with Atrial Fibrillation, and TAVR Thrombosis Management. Curr Cardiol Rep 20: 130.

45. Vora AN, Dai D, Matsuoka R, Harrison JK, Hughes GC, et al. (2018) Incidence, management, and associated clinical outcomes of new-onset atrial fibrillation following transcatheter aortic valve replacement: an analysis from the STS/ACC TVT Registry. JACC Cardiovasc Interv 11(17): 1746-1756.

46. Hui S, Gooley R, Rashid HN, Zaman S (2019) Transcatheter Aortic Valve Replacement and Atrial Fibrillation: Impact of Antithrombotic Strategy on Clinical Outcomes. Heart Lung Circ 28(5): 771-776.

47. Mangner N, Crusius L, Haussig S, Woitek FJ, Kiefer P, et al. (2019) Continued Versus Interrupted Oral Anticoagulation During Transfemoral Transcatheter Aortic Valve Implantation and Impact of Postoperative Anticoagulant Management on Outcome in Patients with Atrial Fibrillation. Am J Cardiol 123(7): 1134-1141.

48. Vimalesvaran K, Dockrill SJ, Gorog DA (2018) Role of rivaroxaban in the management of atrial fibrillation: insights from clinical practice. Vasc Health Risk Manag 14: 13-21.

49. Tarantini G, Mojoli M, Urena M, Vahanian A (2017) Atrial fibrillation in patients undergoing transcatheter aortic valve implantation: epidemiology, timing, predictors, and outcome. Eur Heart J 38(17): 1285-1293.

50. Windecker S, Tijssen J, Giustino G, Guimarães AH, Mehran R, et al. (2017) Trial design: Rivaroxaban for the prevention of major cardiovascular events after transcatheter aortic valve replacement: Rationale and design of the GALILEO study. Am Heart J 184: 81-87.

51. Seeger J, Gonska B, Rodewald C, Rottbauer W, Wöhrle J (2017) Apixaban in Patients with Atrial Fibrillation After Transfemoral Aortic Valve Replacement. JACC Cardiovasc Interv 10(1): 66-74.

52. Collet JP, Berti S, Cequier A, Van Belle E, Lefevre T, et al. (2018) Oral anti-Xa anticoagulation after trans-aortic valve implantation for aortic stenosis: The randomized ATLANTIS trial. Am Heart J 200: 44-50. 